\title{
The cover time of random walks on hypergraphs.
}

\author{
Colin Cooper* $\quad$ Alan Frieze ${ }^{\dagger} \quad$ Thomas Radzik $^{\ddagger}$
}

June 21, 2010

\begin{abstract}
At each step, a random walk moves from its current position on a hypergraph to a randomly selected neighbour. The move is effected by picking a random endpoint of a random hyperedge incident with the current vertex occupied by the walk. We consider two definitions of cover time for random walks on a hypergraph $H$. If the walk sees only the vertices it moves between, then the usual definition of cover time, $C(H)$, applies. If the walk sees the complete edge during the transition, then an alternative definition of cover time, the inform time $I(H)$ is used. We calculate these quantities for random $r$-regular, s-uniform hypergraphs, and find that $C(H) / I(H)=\Omega(s)$ for large $s$. As far as we know, this is the first analysis of cover time for random walks on classes of hypergraphs.

The notion of inform time is a reasonable model of passive listening which fits the following types of situations. The particle is a message transmitted randomly from location to location by a directional transmission in an ad-hoc network, and all receivers within the transmission range can hear. The particle is a rumor passing between friends, which is overheard by other friends present in the group at the same time.
\end{abstract}

\footnotetext{
*Department of Computer Science, King's College, University of London, London WC2R 2LS, UK. e-mail: colin.cooper@kcl.ac.uk

${ }^{\dagger}$ Department of Mathematical Sciences, Carnegie Mellon University, Pittsburgh PA15213, USA. e-mail: alan@random.math.cmu.edu. Supported in part by NSF grant CCF0502793.

${ }^{\ddagger}$ Department of Computer Science, King’s College, University of London, London WC2R 2LS, UK. e-mail: tomasz.radzik@kcl.ac.uk
} 


\section{Introduction}

The problem we consider is intended as a model of the following process. The vertices of a network are associated into groups, and these groups define the edges of the network. In the simplest case, the network is a graph so the groups are exactly the edges of the graph. In general, the groups may be larger, and represent friends, a family, a local computer network, all receivers within transmission range of a directed transmission in an ad-hoc network etc. In this case the network is modeled as a hypergraph, the hyperedges being the group relationships. An individual vertex can be in many groups, and two vertices are neighbours if there share a common hyperedge. Within the network a particle (message, rumor, infection etc) is moving randomly from vertex to neighboring vertex. When this transition occurs all vertices in a given group are somehow affected (infected, informed) by the passage of the particle within the group. Examples of this type of process include the following. The particle is an infection passed from person to person and other family members also become infected with some probability. The particle is a virus traveling on a network connection in an intra-net. The particle is a message transmitted randomly from location to location by a directional transmission in an ad-hoc network, and all receivers within the transmission range can hear. The particle is a rumor passing between friends, which may be overheard by other friends present in the group at the same time.

Let $H=(V(H), E(H))$ be a hypergraph. For $v \in V$ let $d(v)$ be the degree of $v$, i.e. the number of edges $e \in E$ incident with $v$. For $e \in E$ let $s(e)$ be the size $|e|$ of hyperedge $e$, i.e. the number of vertices $v \in e$. Let $N(v)$ be the neighbour set of $v, N(v)=\{w: \exists e \in E, e \supseteq\{v, w\}\}$. We regard $N(v)$ as a multi-set in which each $w \in N(v)$ has a multiplicity equal to the number of edges $e$ containing both $v$ and $w$. Each edge $e \in E$ incident with a vertex $v \in V$ represents a different transmission group. We assume a message $\mathcal{M}$ originated at some vertex $u$ and, at step $t$, is moving randomly from a vertex $v$ to a vertex $w$ in $N(v)$. We model the problem conceptually as a random walk $\mathcal{W}_{u}=\left(W_{u}(0), W_{u}(1), \ldots, W_{u}(t), \ldots\right)$ on the vertex set of hypergraph $H=(V, E)$, where $W_{u}(0)=u$, and $W_{u}(t)=v$.

Several natural models arise for reversible random walks on hypergraphs. Assume that the walk $\mathcal{W}$ is at vertex $v$, and consider the transition from that vertex. In the first model (Model 1 ), an edge $e$ incident with $v$ is chosen proportional to $s(e)-1$, where $s(e)$ is the size of hyperedge $e$. The walk then moves to a random endpoint of that edge, other than $v$. This is equivalent to $v$ choosing a neighbour $w$ uar from the multi-set of neighbours $N(v)$, where vertex $w$ is chosen according to its multiplicity. The stationary distribution of $v$ in Model 1 is given by

$$
\pi_{v}=\frac{\sum_{e: v \in e}(s(e)-1)}{\sum_{e \in E(H)} s(e)(s(e)-1)} .
$$

In the case of graphs this reduces to $\pi_{v}=d(v) / 2 m$, where $d(v)$ is the degree of $v$, and $m$ is the number of edges in the graph. An alternative model (Model 2), is that when $\mathcal{W}$ is at $v$, edge $e$ is chosen uar from the hyperedges incident with $v$, and then $w$ is chosen uar from the 
vertices $w \in e, w \neq v$. The stationary distribution of $v$ in Model 2 is given by

$$
\pi_{v}=\frac{d(v)}{\sum_{u \in V(H)} d(u)},
$$

which corresponds to the familiar formula for graphs. If the hypergraph is uniform (all edges have the same size) then the models are equivalent.

Random walks on graphs are a well studied topic, for an overview see e.g. [1, 10]. Random walks on hypergraphs were used in [5] to cluster together electronic components which are near in graph distance for physical layout in circuit design. For that application, edges were chosen inversely proportional to their size, and then a random vertex within the edge was selected. A random walk model is also used for generalized clustering in [11]. As before, the aim is to partition the edge set, and this is done via the Laplacian of the transition matrix. The only paper we can find which directly considers notions of cover time for random walks on hypergraphs is [3]. The quantity examined in detail in [3] is closer to look-ahead in a random walk on a graph. A look-ahead random walk inspects all neighbours within some fixed distance of the current position.

The (vertex) cover time $C(G)$ of a graph $G$, is given by $C(G)=\max _{u} C_{u}(G)$, where $C_{u}(G)$ is the expected time to visit all vertices of $G$ for a walk starting at vertex $u$. The edge cover time $C_{E}(G)$ is similarly defined as $C_{E}(G)=\max _{u} C_{u, E}(G)$, where $C_{u, E}(G)$ is the expected time to visit all edges of $G$ for a walk starting at vertex $u$.

For a hypergraph $H$, we define three quantities, the vertex cover time $C(H)$, the inform time $I(H)$ and the edge cover time $C_{E}(H)$.

The cover time $C(H)$ of a hypergraph $H$, is given by $C(H)=\max _{u} C_{u}(H)$, where $C_{u}(H)$ is the expected time for the walk $\mathcal{W}_{u}$ described above, to visit all vertices of $H$. To define $I(H)$ we need to define what it means for a vertex to be informed by a step of the walk. Suppose that the walk $\mathcal{W}_{u}$ is at vertex $v$. Using e.g. Model 2, the will first selects an edge $e$ incident with $v$ and then makes a transition to $w \in e$. The vertices of $e$ are said to be informed by this move. The inform time $I(H)$ is the maximum over start vertices $u$, of the expected time at which all vertices of the graph are informed. The edge cover time $C_{E}(H)=\max _{u} C_{u, E}(H)$, where $C_{u, E}(H)$ is the expected time to visit every edge of $H$ for a walk starting at vertex $u$.

For random walks on connected graphs there is an upper bound of $O(n m)$ steps, [2], based on a twice round the spanning tree argument, and there are graphs for which this bound is tight. For Model 1, if we replace each edge $e$ by a graph consisting of a clique of size $\left(\begin{array}{l}e \\ 2\end{array}\right)$ this gives an upper bound of $O\left(n m \overline{s^{2}}\right)$ for connected hypergraphs. Here $\overline{s^{2}}$ is the expected squared edge size $\left(\sum_{e \in E(H)} e^{2}\right) / m$. However, it seems unlikely this is a tight upper bound.

In this paper we analyze random walks for the case of simple random $r$-regular, $s$-uniform hypergraphs $H$. A hypergraph is $r$ regular if each vertex is in $r$ edges, and it is $s$-uniform if every edge is of size $s$. A hypergraph is simple if no edge contains a repeated vertex, and no 
two edges are identical.

Theorem 1. Suppose that $r \geq 2$ and $s \geq 3$ are such that $r s=O(\log n / \log \log n)$. Suppose that $H$ is chosen uar from the set of all simple r-regular, s-uniform hypergraphs with vertex set $V=[n]$. Then whp as $n \rightarrow \infty$,

$$
\begin{gathered}
C(H) \sim\left(1+\frac{1}{(r-1)(s-1)-1}\right) n \log n, \\
I(H) \sim\left(1+\frac{s-1}{(r-1)(s-1)-1}\right) \frac{n}{s-1} \log n,
\end{gathered}
$$

and

$$
C_{E}(H) \sim\left(1+\frac{s-1}{(r-1)(s-1)-1}\right) \frac{r n}{s} \log n .
$$

An interesting case is when $s \rightarrow \infty$, so that $C(H) \sim n \log n$ and

$$
I(H) \sim \frac{r}{r-1} \frac{n}{s} \log n .
$$

Thus, perhaps predictably, inspecting $s$ items at each step leads to an $\Omega(s)$ speed up in cover time.

In the case of graphs, $I(G)=C(G)$, and $C_{E}(G) \geq C(G)$. For hypergraphs, clearly $I(H) \leq$ $C(H)$. However there is the possibility that $C_{E}(H) \leq C(H)$, as every edge can be visited without visiting every vertex. Also, $I(H) \leq C_{E}(H)$ as a vertex is informed whenever the walk covers an edge containing that vertex. Indeed, intuitively we should have $C_{E}(H)$ about $r$ times $I(H)$ if every vertex has degree $r$. We note that our theorem gives $C_{E}(H) \sim r(s-1) / s I(H)$.

\section{Analysis}

\section{$2.1 \quad$ Outline proof of Theorem 1}

We explain the proof of the value of $C(H)$ of Theorem 1; the proofs of $I(H), C_{E}(H)$ are similar. We reduce the walk $\mathcal{W}_{u}(H)$ on the hypergraph $H$ to an equivalent walk $\mathcal{W}_{u}(G)$ on a graph $G(H)$. This is done in Section 2.5. We next establish the conductance of the walk on $G$, and hence the value of the mixing time $T$ on $G$.

The proof of Theorem 1 is based on the following result, which gives the probability that a random walk $\mathcal{W}_{u}(t)$ an a graph $G$, does not visit a given vertex $v$ after a suitably defined mixing time $T$. The result is a corollary of Lemma 3 . 
Lemma 2. Let $\mathcal{A}_{v}(t)$ be the event that a walk $\mathcal{W}_{u}$ on graph $G$, does not visit vertex $v$ at steps $T, T+1, \ldots, t$. Then assuming the conditions of Lemma 3 hold,

$$
\operatorname{Pr}\left(\mathcal{A}_{v}(t)\right)=\frac{\left(1+O\left(T \pi_{v}\right)\right)}{\left(1+p_{v}\right)^{t}}+O\left(T^{2} \pi_{v} e^{-t / K T}\right),
$$

where $p_{v}$ is given by (9), and $K$ is a large constant.

The value of $p_{v}$, as defined in (9) is given by $p_{v} \sim \pi_{v} / R_{v}$, where $\pi_{v}$ is the stationary distribution of $v$, and $R_{v}$ can be calculated from the structure of the graph around $v$. The precise definitions of $T, R_{v}$ are given in Section 2.2 below.

In Section 2.4 we define a property, tree-like vertex of a hypergraph, which holds for most vertices of $H$, whp. In Section 3, we derive the parameter $p_{v}$ of the walk in the associated graph $G$, for tree-like vertices $v$ of $H$. In Section we prove the conditions of Lemma 3 hold for the associated graph $G$, provided $v$ is tree-like. Having established this, we can apply Lemma 2 to such vertices. In Section 4 we prove Theorem 1. The proof is given in most detail for $C(H)$. An upper bound on $C(H)$ is established in Section 4.1, and a lower bound in Section 4.2 .

\subsection{First visit probability}

Let $G$ denote a fixed connected graph with $n$ vertices and $m$ edges. Let $P$ be the matrix of transition probabilities of the walk and let $P_{u}^{(t)}(v)=\operatorname{Pr}\left(W_{u}(t)=v\right)$. We assume the random walk $\mathcal{W}_{u}$ on $G$ is ergodic, and thus the random walk has stationary distribution $\pi$, where $\pi_{v}=d(v) /(2 m) . d(v)$ is the degree of vertex $v$.

In this section we consider the distribution of the number of visits to a given vertex $v$ made by a random walk $\mathcal{W}_{u}(t)$. We use the approach developed in [6], [7].

Let $\lambda_{2}$ be the second eigenvalue of $P$, and let $\Phi_{G}$ be the conductance of $G$ i.e. $\Phi_{G}=$ $\min _{S \subseteq V, \pi_{S} \leq 1 / 2} \Phi_{G}(S)$ where

$$
\Phi_{G}(S)=\frac{\sum_{x \in S} \pi_{x} P(x, \bar{S})}{\pi_{S}} .
$$

Then,

$$
\begin{aligned}
& 1-\Phi_{G} \leq \lambda_{2} \leq 1-\frac{\Phi_{G}^{2}}{2} \\
& \left|P_{u}^{(t)}(x)-\pi_{x}\right| \leq\left(\pi_{x} / \pi_{u}\right)^{1 / 2} \lambda_{2}^{t} .
\end{aligned}
$$

A proof of this can be found for example in Jerrum and Sinclair [9]. (For (4) we need $\lambda_{2}=\lambda_{\max }$ which can be achieved by making the chain lazy i.e. by not moving with probability $1 / 2$ at each step. This has no significant effect on the analysis). 
Let $T$ be such that, for $t \geq T$

$$
\max _{u, x \in V}\left|P_{u}^{(t)}(x)-\pi_{x}\right| \leq n^{-3} .
$$

If this inequality holds, we say the distribution of the walk is in near stationarity. Fix two vertices $u, v$. We consider the returns to vertex $v$ made by a walk $\mathcal{W}_{v}$, starting at $v$. Let $r_{t}=\operatorname{Pr}\left(W_{v}(t)=v\right)$ be the probability that the walk returns to $v$ at step $t=0,1, \ldots$ In particular note that $r_{0}=1$, as the walk starts on $v$.

Let

$$
R_{T}(z)=\sum_{j=0}^{T-1} r_{j} z^{j} .
$$

Thus, evaluating $R_{T}(z)$ at $z=1$, we have $R_{T}(1) \geq r_{0}=1$.

For $t \geq T$ let $f_{t}=f_{t}(u \rightarrow v)$ be the probability that the first visit made to $v$ by the walk $\mathcal{W}_{u}$ to $v$ in the period $[T, T+1, \ldots]$ occurs at step $t$.

The following lemma gives the probability that a walk, starting from near stationarity makes a first visit to vertex $v$ at a given step. For proofs of the lemma and its corollary, see [6], [7].

Lemma 3. Let $R_{v}=R_{T}(1)$, where $R_{T}(z)$ is from (6). For some sufficiently large constant $K$, let

$$
\lambda=\frac{1}{K T},
$$

where T satisfies (5). Suppose that

(i) For some constant $\theta>0$, we have

$$
\min _{|z| \leq 1+\lambda}\left|R_{T}(z)\right| \geq \theta .
$$

(ii) $T \pi_{v}=o(1)$ and $T \pi_{v}=\Omega\left(n^{-2}\right)$.

There exists

$$
p_{v}=\frac{\pi_{v}}{R_{v}\left(1+O\left(T \pi_{v}\right)\right)},
$$

such that for all $t \geq T$,

$$
f_{t}(u \rightarrow v)=\left(1+O\left(T \pi_{v}\right)\right) \frac{p_{v}}{\left(1+p_{v}\right)^{t+1}}+O\left(T \pi_{v} e^{-\lambda t / 2}\right) .
$$




\subsection{Configuration model}

We will need a workable model of an $r$-regular $s$-uniform hypergraph. We use a hypergraph version of the configuration model of Bollobás [4]. A configuration $C(r, s)$ consists of a partition of $r n$ labeled points $\left\{a_{1,1}, \ldots, a_{1, r}, \cdots, a_{n, 1}, \ldots, a_{n, r}\right\}$ into unordered sets $E_{i}, i=1, \ldots, r n / s$ of size $s$. We assume naturally that $s$ divides $r n$. We refer to these sets as the hyperedges of the configuration, and to the sets $v_{i}=\left\{a_{i, 1}, \ldots, a_{i, r}\right\}$ as the vertices. By identifying the points of $v_{i}$, we obtain an $r$-regular, s-uniform (multi-)hypergraph $H(C)$. In general, many configurations map to one underlying hypergraph $H(C)$. Considering the set $\mathcal{C}(r, s)$ of all configurations $C(r, s)$ with the uniform measure, the measure $\mu(H(C))$ depends only on the number of parallel edges (if any) at each vertex, and as an example all simple hypergraphs i.e. those without multiple edges have equal measure in $H(C)$. The probability a uar sampled configuration is graphic is bounded below by a constant dependent only on $r$ and $s$.

For the values of $r, s$ considered in this paper, the probability $H(C)$ is simple is $\Omega\left(e^{-(r-1)(s-1)}\right)$. It follows that any almost sure property of $H(C)$ is also an almost sure property of simple hypergraphs $H$.

\subsection{Tree-like vertices}

We need to identify the local structure of a typical vertex of $H$. Let

$$
\omega=\log \log \log n .
$$

A sequence $v_{1}, v_{2}, \ldots, v_{k} \in V$ is said to define a path of length $k-1$ if there are distinct edges $e_{1}, e_{2}, \ldots, e_{k-1} \in E$ such that $\left\{v_{i}, v_{i+1}\right\} \subseteq e_{i}$ for $1 \leq i \leq k-1$. A sequence $v_{1}, v_{2}, \ldots, v_{k} \in$ $V, k \geq 3$ is said to define a cycle of length $k$ if there are distinct edges $e_{1}, e_{2}, \ldots, e_{k} \in E$ such that $\left\{v_{i}, v_{i+1}\right\} \subseteq e_{i}$ for $1 \leq i \leq k$. (Here $v_{k+1}=v_{1}$ ). A path/cycle is small if it has length at most $\omega$. A vertex $v \in V$ is said to be locally-tree-like if there does not exist a small path $v=v_{1}, v_{2}, \ldots, v_{k}$ from $v$ to a small cycle $v_{k}=w_{1}, w_{2}, \ldots, w_{l}$. An edge is locally-tree-like if it only contains tree-like vertices.

We argue next that almost all vertices of $H$ are locally-tree-like.

Lemma 4 . Whp there are at most $(r s)^{3 \omega}$ vertices that are not locally-tree-like.

Proof We work with the model $H(C)$. The expected number of vertices on cycles of length $k$ can be bounded above by

$$
\sum_{k=3}^{\omega} s k n^{k}\left(\frac{r s}{n}\right)^{k} \leq O\left(s \omega(r s)^{\omega}\right) .
$$

The Markov inequality implies that whp there are at most $(r s)^{2 \omega}$ vertices on small cycles. For each such vertex there are at most $(r s)^{\omega}$ vertices reachable by a walk of length $\omega$. 
Lemma 5. Whp there are no short walks joining distinct short cycles.

Proof If such a structure exists then there exists a walk $v_{1}, v_{2}, \ldots, v_{k}$ of length at most $3 \omega$ and a pair $i, j \in[k]$ and edges $f_{1}, f_{2} \in E$ such that $v_{1}, v_{i} \in f_{1}$ and $v_{k}, v_{j} \in f_{2}$. The probability of this is at most

$$
\sum_{k=5}^{3 \omega} s^{2} k^{2} n^{k}\left(\frac{r s}{n}\right)^{k+1}=O\left(\frac{s^{3} \omega^{3}(r s)^{3 \omega}}{n}\right)
$$

\subsection{Construction of an equivalent (contracted) graph}

To calculate the quantities $C(H), I(H)$ and $C_{E}(H)$ we replace $H$ by graphs $G(H), \Gamma(v)$ and $\Gamma(e)$, where $v, e$ are a tree-like vertex and edge of $H$ respectively. The precise construction of these graphs is as follows:

Clique graph $G(H)$. To estimate the cover time of hypergraph $H$ we define a (multi-)graph $G(H)$ with the same cover time. To obtain $G(H)$ we replace each hyperedge $e(H)$ by a clique of size $|e(H)|$, transforming the hypergraph $H$ into a multi-graph $G(H)$, which we call the clique graph of $H$. Formally, $G(H)=(V, F)$ where $F=\bigcup_{e \in E}\left(\begin{array}{l}e \\ 2\end{array}\right)$.

We can think of $\mathcal{W}_{u}$ as a walk on $G(H)$. Thus, the cover time of $H$ is the cover time of $G(H)$.

Inform-Contraction graph $\Gamma(v)$. Let $S_{v}$ be the multi-set of edges $\{w, x\}$ in $G(H)$, not containing $v$, but which are contained in hyperedges incident with vertex $v$ in $H$ i.e.

$$
S_{v}=\{\{w, x\}: \exists e \in E, v \in e, \text { and } w, x \in e \backslash\{v\}\} .
$$

Since $H$ is $r$-regular and $s$-uniform, each $S_{v}$ has size $r\left(\begin{array}{c}s-1 \\ 2\end{array}\right)$.

A vertex $v$ is informed if either (i) $v$ is visited or (ii) $S_{v}$ is visited by $\mathcal{W}_{u}$. To compute the probability that $v$ or $S_{v}$ is visited we subdivide each edge $f=\{w, x\}$ of $S_{v}$ by introducing an artificial vertex $a_{f}$. Thus $f$ is replaced by $\left\{w, a_{f}\right\},\left\{a_{f}, x\right\}$. Call the resulting graph $G_{v}(H)$. Let $D_{v}=\{v\} \cup\left\{a_{f}: f \in S_{v}\right\}$ and note that $D_{v}$ is independent in $G_{v}(H)$. Now contract $D_{v}$ to a single vertex $\gamma=\gamma\left(D_{v}\right)$. Let $\Gamma(v)$ be the resulting multi-graph. The degree of $\gamma$ is then

$$
d(\gamma)=r\left(2\left(\begin{array}{c}
s-1 \\
2
\end{array}\right)+(s-1)\right)=r(s-1)^{2} .
$$

Furthermore,

$$
d(\Gamma(v))=r(s-1) n+r(s-1)(s-2) .
$$


For a random walk in $\Gamma$ the stationary distribution of $\gamma$ is thus

$$
\pi(\gamma)=\frac{s-1}{n+s-2}
$$

Note that $m_{v}=r(s-1) n / 2+r\left(\begin{array}{c}s-1 \\ 2\end{array}\right)=\mid E\left(\Gamma(v)|=| E\left(G(H) \mid+r\left(\begin{array}{c}s-1 \\ 2\end{array}\right)\right.\right.$.

Suppose now that $\mathcal{X}_{u}$ is a random walk in $G_{v}(H)$ starting at $u \notin D_{v}$. For $t \geq T$, let $\mathcal{B}_{v}(t)$ be the event that the walk $\mathcal{W}_{u}$ in $G(H)$ does not visit $S_{v} \cup\{v\}$ at steps $T, T+1, \ldots, t$. Then $\mathcal{B}_{v}(t)$ is equivalent to $\wedge_{x \in D_{v}} \mathcal{A}_{x}(t)$ defined with respect to $\mathcal{X}_{u}$.

Edge-Contraction graph $\Gamma(e)$. Starting from $G(H)$, and given $e \in E(H)$ form $G_{e}(H)$ as follows. For each of the edges $f=\{u, v\} \in\left(\begin{array}{l}e \\ 2\end{array}\right)$, subdivide $f$ using an artificial vertex $a_{f}$. Thus $f$ is replaced by $\left\{u, a_{f}\right\},\left\{a_{f}, v\right\}$. The set $D_{e}=\left\{a_{f}: f \in e \in H\right\}$ gives rise to $G_{e}(H)$ as above. Contract $D_{e}$ to a vertex $\gamma$ to form a graph $\Gamma(e)$, as for $\Gamma(v)$.

The degree of $\gamma$ is then

$$
d(\gamma)=s(s-1)
$$

Furthermore,

$$
d(\Gamma(e))=r n(s-1)+\left(\begin{array}{l}
s \\
2
\end{array}\right) .
$$

For a random walk in $\Gamma$ the stationary distribution of $\gamma$ is thus

$$
\pi(\gamma)=\frac{2 s}{2 r n+s}
$$

Suppose now that $\widehat{\mathcal{X}}_{u}$ is a random walk in $G_{e}(H)$ starting at $u \notin D_{e}$. For $t \geq T$, let $\mathcal{B}_{e}(t)$ be the event that the walk $\mathcal{W}_{u}$ in $G_{e}(H)$ does not visit $D_{e}$ at steps $T, T+1, \ldots, t$.

Lemma 6. Let $x=v$, e. Let $\Gamma=\Gamma(v), \Gamma(e)$. Let $\mathcal{Y}_{u}$ be a random walk in $\Gamma$ starting at $u \neq \gamma$. Let $T$ be a mixing time satisfying $(5)$ in both $G_{x}(H)$ and $\Gamma$. Then

$$
\operatorname{Pr}\left(\mathcal{A}_{\gamma}(t) ; \Gamma\right)=\operatorname{Pr}\left(\mathcal{B}_{x}(t) ; G(H)\right)\left(1+O\left(\frac{s}{n}\right)\right)
$$

where the probabilities are those derived from the walk in the given graph.

\section{Proof}

We give the proof for $\Gamma(v)$. The proof for $\Gamma(e)$ is similar. Let $Y_{x}(j)$ (resp. $X_{x}(j)$ ) be the position of walk $\mathcal{Y}_{x}\left(\operatorname{resp} . \mathcal{X}_{x}(j)\right)$ at step $j$. Let $\Lambda=G(H), \Gamma(v)$ and let $P_{u}^{s}(x ; \Lambda)$ be the 
transition probability in $\Lambda$, for the walk to go from $u$ to $x$ in $s$ steps.

$$
\begin{aligned}
\operatorname{Pr}\left(\mathcal{A}_{\gamma}(t) ; \Gamma(v)\right) & =\sum_{y \neq \gamma} P_{u}^{T}(y ; \Gamma(v)) \operatorname{Pr}\left(Y_{y}(\sigma-T) \neq \gamma, T \leq \sigma \leq t ; \Gamma(v)\right) \\
& =\sum_{y \neq \gamma}\left(\frac{d(y)}{2 m}\left(1+O\left(n^{-3}\right)\right)\right) \operatorname{Pr}\left(Y_{y}(\sigma-T) \neq \gamma, T \leq \sigma \leq t ; \Gamma(v)\right) \\
& =\sum_{x \notin D_{v}}\left(P_{u}^{T}\left(x ; G_{v}(H)\right)(1+O(s / n))\right) \operatorname{Pr}\left(X_{x}(\sigma-T) \notin D_{v}, T \leq \sigma \leq t ; G_{v}(H)\right) \\
& =\operatorname{Pr}\left(\wedge_{x \in D_{v}} \mathcal{A}_{x}(t) ; G_{v}(H)\right)(1+O(s / n))
\end{aligned}
$$

Equation (13) follows from (5). Equation (14) follows because there is a natural measure preserving map $\phi$ between walks in $G_{v}(H)$ that start at $x \notin D_{v}$ and avoid $D_{v}$ and walks in $\Gamma(v)$ that start at $x \neq \gamma$ and avoid $\gamma$.

To establish the theorem we need precise estimates of $R_{v}$ in $G(H)$ and $R_{\gamma}$ in $\Gamma(v)$ (resp. $\Gamma(e)$ ). Once this is done the theorem will follow from Corollary 2. But first we have to estimate the value of $T$ in (5).

\subsection{Mixing time of the random walk}

We estimate the conductance of the walks on $G(H)$ and $\Gamma(v)$.

\subsubsection{Conductance of $G(H)$}

Lemma 7. Suppose that $r=2, s \geq 3$ or that $r \geq 3$. Let $C(r, s)$ be sampled uar from $\mathcal{C}(r, s)$. Then there exists $\epsilon>0$ such that whp there is no set of $t \leq n / 2$ vertices that contain $r t(1-\epsilon) / s$ edges or more.

Proof Let $N(t, k, r, s)$ be the expected number of sets of configuration vertices of size $t$ which induce at least $k$ hyperedges, where $k s=r t(1-\epsilon)$. We will prove the lemma by showing that in the configuration model

$$
\sum_{t=1}^{n / 2} N(t, k, r, s)=o\left(n^{-1 / 10}\right)
$$

Now

$$
N(t, k, r, s) \leq\left(\begin{array}{l}
n \\
t
\end{array}\right)\left(\begin{array}{l}
r t \\
k s
\end{array}\right) \frac{F(k s) F(r n-k s)}{F(r n)}
$$


where $F(a)=a ! /\left((a / s) !(s !)^{(a / s)}\right)$ for $s \mid a$. Note that if $s \mid a, b$ and $a>b$ then

$$
\frac{F(b) F(a-b)}{F(a)}=\frac{\left(\begin{array}{l}
a / s \\
b / s
\end{array}\right)}{\left(\begin{array}{l}
a \\
b
\end{array}\right)}=O(\sqrt{s})\left(\frac{b}{a}\right)^{b(s-1) / s}\left(1-\frac{b}{a}\right)^{(a-b)(s-1) / s}
$$

and that

$$
\begin{aligned}
\left(\begin{array}{l}
n \\
t
\end{array}\right) & =O(1) \sqrt{\frac{n}{t(n-t)}}\left(\frac{n}{t}\right)^{t}\left(1-\frac{t}{n}\right)^{t-n} \\
\left(\begin{array}{l}
r t \\
k s
\end{array}\right) & =O(1) \sqrt{\frac{r t}{k s(r t-k s)}}\left(\frac{r t}{k s}\right)^{k s}\left(1-\frac{k s}{r t}\right)^{k s-r t} .
\end{aligned}
$$

Thus, assuming $t \leq n / 2$ and that $k s=r t(1-\epsilon)$ where $\epsilon>0$ constant,

$$
\begin{aligned}
N(t, k, r, s)= & O\left(\frac{1}{\sqrt{k s}}\right)\left(\frac{r n}{k s}\right)^{k}\left(\frac{n}{t}\right)^{t-k s}\left(1-\frac{k s}{r n}\right)^{(r n-k s)(s-1) / s}\left(1-\frac{k s}{r t}\right)^{k s-r t}\left(1-\frac{t}{n}\right)^{t-n} \\
= & O(1)\left(\frac{n}{(1-\epsilon) t}\right)^{r t(1-\epsilon) / s}\left(\frac{t}{n}\right)^{r t(1-\epsilon)-t}\left(\frac{1}{\epsilon}\right)^{\epsilon r t}\left(1-\frac{t(1-\epsilon)}{n}\right)^{r(n-t(1-\epsilon)) \frac{s-1}{s}}\left(1-\frac{t}{n}\right)^{t-n} \\
= & O(1)\left(\left(\frac{1}{1-\epsilon}\right)^{(1-\epsilon) / s}\left(\frac{1}{\epsilon}\right)^{\epsilon}\left(1-\frac{t(1-\epsilon)}{n}\right)^{\epsilon(1-1 / s)}\left(\frac{t}{n}\right)^{(1-\epsilon)(1-1 / s)-1 / r}\right)^{t r} \\
& \times\left(\frac{(1-t(1-\epsilon) / n)^{r(1-1 / s)}}{1-t / n}\right)^{n-t}
\end{aligned}
$$

To establish an upper bound, we first consider the term (17). We write

$$
\begin{aligned}
\frac{(1-t(1-\epsilon) / n)^{r(1-1 / s)}}{1-t / n} & =\left(1+\frac{\epsilon t}{n-t}\right)^{r(1-1 / s)}\left(1-\frac{t}{n}\right)^{r(1-1 / s)-1} \\
& \leq \exp \left\{\frac{t}{n}(1-r(1-1 / s)(1-2 \epsilon)\}\right.
\end{aligned}
$$

Now $r(1-1 / s) \geq 4 / 3$ and so if $\epsilon<1 / 8$ we find that the contribution of (17) is less than one. Considering (16), for $t \leq n / 2$ it holds that $t / n \leq 1-t(1-\epsilon) / n$, and thus (16) is $O\left(\Psi_{t}^{t r}\right)$ where $\Psi_{t}$ is given by

$$
\Psi_{t}=\left(\frac{1}{1-\epsilon}\right)^{(1-\epsilon) / s}\left(\frac{1}{\epsilon}\right)^{\epsilon}\left(\frac{t}{n}\right)^{(1-2 \epsilon)(1-1 / s)-1 / r} .
$$

Provided $(1-2 \epsilon)(1-1 / s)-1 / r>0, \Psi$ is monotone increasing in $t$, and putting $t=n / 2, r=$ $2, s=3$,

$$
\Psi_{t} \leq\left(\frac{1}{1-\epsilon}\right)^{(1-\epsilon) / 3}\left(\frac{1}{\epsilon}\right)^{\epsilon}\left(\frac{1}{2}\right)^{(1-2 \epsilon)(2 / 3)-1 / 2}
$$


and choosing $\epsilon=1 / 100$ we find $\Psi_{t}<0.95$. Thus

$$
\sum_{t=1}^{n / 2} N(t, k, r, s) \leq \sum_{t=1}^{n / 2} \Psi_{t}^{t r}=o\left(n^{-1 / 10}\right)
$$

Going back to (2) we see that if $G$ is a $d$-regular graph then $\Phi_{G}(S)=\frac{e(S: \bar{S})}{d|S|}$, were $e(S: \bar{S})$ denotes the number of edges with one endpoint in $S$ and the other in $\bar{S}=V \backslash S$. Note that in this case $\pi(S) \leq 1 / 2$ if $|S| \leq n / 2$.

The corollary below follows from Lemma 7, and the definition of the underlying clique graph $G(H)$ of a hypergraph $H$.

Corollary 8. The conductance $\Phi_{G}$ of the graph $G(H)$ is $\Omega(1 / s)$ whp.

Proof $\quad$ For $S \subseteq V,|S|=t \leq n / 2$,

$$
\Phi_{G}(S)=\frac{e(S: \bar{S})}{d|S|} \geq \frac{(s-1) \epsilon r t}{\left(\begin{array}{l}
s \\
2
\end{array}\right) r t}=\Omega(1 / s) .
$$

Corollary 9. The conductance $\Phi_{\Gamma}$ of the graph $\Gamma=\Gamma(v), \Gamma(e)$ is $\Omega(1 / s)$ whp.

Proof Note first that contracting vertices cannot reduce conductance. This is because we minimise the same $\Phi(S)$ value over a smaller collection of sets $S$. It is a simple matter to see that subdividing at most $r\left(\begin{array}{l}s \\ 2\end{array}\right)$ edges within $S$ increases the degree of $S$ by at most $r s(s-1)$ and thus $\Phi_{\Gamma}=\Omega(1 / s)$.

It follows from (3) and (4) that in both $G(H)$ and $\Gamma(v), \Gamma(e)$, we can take $T=O\left(s^{2} \log n\right)=$ $o\left(\log ^{3} n\right)$ in $(5)$.

\section{$3 \quad$ To apply Lemma 2}

\subsection{Returns in $G(H)$}

For a vertex $v$ and integer $k \geq 1$ let $N_{k}(v)$ denote the set of vertices $w$ for which there is a path of length at most $k$ from $v$ to $w$. The following construction models an infinite extension of the neighbourhood of $v$ in $G=G(H)$, for a locally-tree-like vertex $v$. Let $\mathcal{T}_{G}^{*}$ be an infinite graph (with a tree-like structure) defined recursively as a root $h$ joined to each vertex of $r-1$ disjoint cliques $C_{1}, C_{2}, \ldots, C_{r-1}$ of size $s-1$. Each vertex in $C_{1} \cup \cdots \cup C_{r}$ is the root of a 
further disjoint copy of $\mathcal{T}_{G}^{*}$. For $\mathcal{T}_{G}$ we take a root vertex $h$ and join it to each vertex of $r$ disjoint cliques $C_{1}, C_{2}, \ldots, C_{r}$ of size $s-1$. Each vertex in $C_{1} \cup \cdots \cup C_{r}$ is the root of a further disjoint copy of $\mathcal{T}_{G}^{*}$. If $v$ is locally-tree-like, then provided $k \leq \omega$, the subgraph of $G(H)$ induced by $N_{k}(v)$ is isomorphic to the first $k$ levels of $\mathcal{T}_{G}$.

We first compute the expected number of returns $R_{G}$ to the root for a random walk on $\mathcal{T}_{G}$. We can then argue as in the proof of Lemma 7 of [6] that $R_{v}=R_{G}+o(1)$ for a locally-tree-like vertex $v$ of $G(H)$. We can project a walk on $\mathcal{T}_{G}$ onto the non-negative integers by mapping a vertex $v$ of $\mathcal{T}_{G}$ to its distance $\Delta_{v}$ from the root. If $v \neq h$ then $v$ has degree $(s-1) r$, and if the walk is at $v$, then it moves to a neighbour $w$ where

$$
\Delta_{w}= \begin{cases}\Delta_{v}+1 & \text { probability } \frac{r-1}{r} \\ \Delta_{v} & \text { probability } \frac{s-2}{r(s-1)} \\ \Delta_{v}-1 & \text { probability } \frac{1}{r(s-1)}\end{cases}
$$

Now $R_{G}$ is the expected number of returns to the origin of a random walk on the non-negative integers, with probabilities defined as in (19).

We note the following result (see e.g. [8]), for a random walk on the non-negative integers $\{0,1, \ldots\}$ with transition probabilities at $k>0$ of $q<p$ for moves left and right respectively. Starting at vertex 1 , the probability of ultimate return to the origin 0 is

$$
\rho=\frac{q}{p} .
$$

It follows that if the walk always moves to 1 from the origin than the expected number of returns $R$ to the origin is given by

$$
R=\frac{1}{1-\rho}=\frac{p}{p-q} .
$$

In which case we see that

$$
R_{v}=R_{G}+o(1)=\frac{(r-1)(s-1)}{(r-1)(s-1)-1}+o(1) .
$$

Finally, for locally-tree-like vertices $v$ we have that the value of $p_{v}$ in (9) is given by

$$
p_{v}=(1+o(1)) \frac{1}{n} \frac{(r-1)(s-1)-1}{(r-1)(s-1)} .
$$

\subsection{Returns in $\Gamma(v)$}

The following construction models an infinite extension of the neighbourhood of $\gamma$ in $\Gamma=$ $\Gamma(v)$, for a locally-tree-like vertex $v$. Let $\mathcal{T}_{\Gamma}$ be an infinite multi-graph consisting of a root $h$ 
(corresponding to $\gamma$ ) joined to $r(s-1)$ distinct vertices $w_{i, j}, i=1,2, \ldots, r, j=1,2, \ldots s-1$ (corresponding to the vertices in cliques with $v$ ) and with $s-1$ parallel edges between $h$ and each $w_{i, j}$. Each vertex $w=w_{i, j}$ is the root of $r-1$ copies of an infinite tree isomorphic to $\mathcal{T}_{G}^{*}$ defined in Section 3.1.

The probability $P_{\gamma}$ of a return to $h$ of a walk on $\mathcal{T}_{\Gamma}$ starting at $h$ is given by

$$
P_{\gamma}=\sum_{k=0}^{\infty} \rho^{k}(1-\hat{\rho})^{k} \hat{\rho}=\frac{\hat{\rho}}{1-\rho(1-\hat{\rho})}
$$

where $\rho=\frac{1}{(r-1)(s-1)}($ see $(19)$ and $(20))$ is the probability of a return to the root $w$ of a $\mathcal{T}_{G}^{*}$ and $\hat{\rho}=\frac{s-1}{s-1+(r-1)(s-1)}=\frac{1}{r}$ is the probability of moving from a $w_{i, j}$ to the root $h$ in a single step. Plugging these values into (23) gives

$$
P_{\gamma}=\frac{s-1}{r(s-1)-1} .
$$

Therefore, using arguments similar to those in Lemma 7 of [6] we see that

$$
R_{\gamma}=\frac{1}{1-P_{\gamma}}+o(1)=\frac{r(s-1)-1}{(r-1)(s-1)-1}+o(1) .
$$

For locally-tree-like vertices $v$, using (11), the value of $p_{\gamma}$ in (9) is given by

$$
p_{\gamma}=(1+o(1)) \frac{s-1}{n} \frac{(r-1)(s-1)-1}{r(s-1)-1} .
$$

\subsection{Returns in $\Gamma(e)$}

Let $\mathcal{T}_{\Gamma}^{\prime}$ be an infinite multi-graph consisting of a root $h$ (corresponding to $\gamma$ ) joined to $s$ distinct vertices $w_{i}, i=1,2, \ldots s$ (corresponding to the vertices in clique of edge $e$ ) and with $s-1$ parallel edges between $h$ and each $w_{i}$. Each vertex $w=w_{i}$ is the root of $r-1$ copies of an infinite tree isomorphic to $\mathcal{T}_{G}^{*}$ defined in Section 3.1.

We find $R_{\gamma}^{\prime}=(1+o(1)) R_{\gamma}$, that $d(\gamma)=s(s-1)$ and that $d(\Gamma(e))=r n(s-1)+\left(\begin{array}{l}s \\ 2\end{array}\right)$.

For locally-tree-like vertices $v$, using (12), and assuming $s=o(n)$, the value of $p_{\gamma}$ in (9) is given by

$$
p_{\gamma}=(1+o(1)) \frac{s}{r n} \frac{(r-1)(s-1)-1}{r(s-1)-1} .
$$




\subsection{Technical condition (8)}

We will only verify this for locally-tree-like vertices. Observe first that if $R=R_{v}, R_{\gamma}$ satisfies $R \leq 2-\epsilon$ for some constant $\epsilon>0$ then it is easy to verify this condition. Indeed, for $|z| \leq 1+\lambda$,

$$
\left|R_{T}(z)\right| \geq r_{0}-(1+\lambda)^{T} \sum_{t=1}^{T} r_{t}=1-(1+\lambda)^{T}(R-1) \geq 1-(1+\lambda)^{T}(1-\epsilon)>\epsilon / 2 .
$$

\subsubsection{Case of $G(H)$}

We write

$$
R_{v}=1+\frac{1}{(r-1)(s-1)-1}+o(1)
$$

and see that we only need to consider $r=2, s=3$.

For any $z$,

$$
\left|R_{T}(z)-R_{T}(1)\right| \leq \sum_{j=1}^{T} r_{j}\left|z^{j}-1\right| .
$$

Now $R_{v} \sim 2$ in our case, so we only need to show that the RHS of (27) is strictly less than 2.

Next observe that $\pi_{v}=1 / n$ for $v \in V$ and that (4) implies that

$$
S_{0}=\sum_{i=\omega}^{T} r_{j}\left|z^{j}-1\right| \leq 2 \sum_{i=\omega}^{T} r_{i} \leq 2 \sum_{i=\omega}^{T}\left(\lambda_{\max }^{i}+\pi_{v}\right)=o(1)
$$

since $\lambda_{\max } \leq \zeta<1$ for some constant $\zeta$. This follows from (3).

Now consider $j<\omega$. Fix $0 \leq \theta<2 \pi$ and let $z=e^{i \theta}$, then

$$
\left|z^{j}-1\right|=(2(1-\cos j \theta))^{1 / 2}=2|\sin j \theta / 2| .
$$

Then

$$
S_{1}=\sum_{j=1}^{\omega-1} r_{j}\left|z^{j}-1\right|=\sum_{j=1}^{\omega-1} r_{j}(2(1-\cos j \theta))^{1 / 2}=2 \sum_{j=1}^{\omega-1} r_{j}|\sin j \theta / 2| .
$$

Note that $r_{1}=0, r_{2}=\frac{1}{4}$ and $r_{3}=\frac{1}{16}$. Suppose first that $\theta \notin I=\left[\frac{3 \pi}{8}, \frac{5 \pi}{8}\right] \cup\left[\frac{11 \pi}{8}, \frac{13 \pi}{8}\right]$. Then $|\sin \theta| \leq \sin \frac{3 \pi}{8}$ and so

$$
S_{1} \leq 2 \sum_{j=1}^{\omega-1} r_{j}-r_{2}\left(1-\sin \frac{3 \pi}{8}\right)
$$


On the other hand, if $\theta \in I$ then $|\sin 3 \theta / 2| \leq \sin \frac{7 \pi}{16}$ and then

$$
S_{1} \leq 2 \sum_{j=1}^{\omega-1} r_{j}-r_{3}\left(1-\sin \frac{7 \pi}{16}\right)
$$

From (4),

$$
R_{T}(1)=1+\sum_{j=1}^{\omega-1} r_{j}+O\left(\lambda_{2}^{\omega}\right) .
$$

Thus, as $R_{T}(1)=2+o(1)$, (28), (29), (30) imply that $S_{0}+S_{1} \leq 2\left(R_{T}(1)-1\right)-1 / 20=$ $39 / 20-o(1)$.

This confirms that the RHS of (27) is less than 2 and that technical condition (8) holds.

\subsubsection{Case of $\Gamma(v)$}

From (24) we write

$$
R_{\gamma}=1+\frac{s-1}{(r-1)(s-1)-1}+o(1)
$$

Once again we see that we only need to consider $r=2, s=3$.

We then use the same argument as in Section 3.4.1. Equation (29) holds and this time $r_{2}=1 / 2, r_{3}=0, r_{4}=1 / 3$. If $\theta \in I$ then $|\sin 2 \theta| \leq \sin \frac{\pi}{4}$ and then

$$
S_{1} \leq 2 \sum_{j=1}^{\sigma-1} r_{j}-r_{4}\left(1-\sin \frac{\pi}{4}\right)
$$

We use (31) in place of (30) to prove that technical condition (8) holds.

\subsubsection{Case of $\Gamma(e)$}

It was noted in Section 3.3 that the value of $R_{\gamma}^{\prime}$ in $\Gamma(e)$ satisfies $R_{\gamma}^{\prime}=(1+o(1)) R_{\gamma}$ in $\Gamma(v)$, as given in (24). Thus the results of the above section apply.

\section{Cover time of $G(H)$}

We follow a similar argument to that given in [6]. 


\subsection{Upper bound on cover time}

Let $t_{0}=(1+o(1)) \frac{(r-1)(s-1)}{(r-1)(s-1)-1} n \log n$ where the $o(1)$ term is large enough so that all inequalities below are satsified. Let $T_{G}(u)$ be the time taken to visit every vertex of $G$ by the random walk $\mathcal{W}_{u}$. Let $U_{t}$ be the number of vertices of $G=G(H)$ which are not visited by $\mathcal{W}_{u}$ in the interval $[T, t]$. We note the following:

$$
\begin{aligned}
C_{u}=C_{u}(H)=\mathbf{E} T_{G}(u) & =\sum_{t>0} \operatorname{Pr}\left(T_{G}(u)>t\right), \\
\operatorname{Pr}\left(T_{G}(u)>t\right) & =\operatorname{Pr}\left(U_{t}>0\right) \leq \min \left\{1, \mathbf{E} U_{t}\right\} .
\end{aligned}
$$

It follows from (32), (33) that for all $t$

$$
C_{u} \leq t+\sum_{\sigma \geq t} \mathbf{E} U_{\sigma}=t+\sum_{v \in V} \sum_{\sigma \geq t} \operatorname{Pr}\left(\mathcal{A}_{v}(\sigma)\right) .
$$

Let $V_{1}$ be the set of locally-tree-like vertices and let $V_{2}=V-V_{1}$.

We apply Corollary 2. For $v \in V_{1}$, from (22) we have $n p_{v} \sim \frac{(r-1)(s-1)-1}{(r-1)(s-1)}$. Hence,

$$
\begin{aligned}
\sum_{\sigma \geq t_{0}} \operatorname{Pr}\left(\mathcal{A}_{v}(\sigma)\right) & \leq(1+o(1)) e^{-t_{0} p_{v}} \sum_{\sigma \geq t_{0}} e^{-\left(\sigma-t_{0}\right) p_{v}}+O\left(e^{-\lambda t_{0} / 2}\right) \\
& \leq 2 p_{v}^{-1} e^{-t_{0} p_{v}} \\
& \leq 5
\end{aligned}
$$

Furthermore, we see also that,

$$
\operatorname{Pr}\left(\mathcal{A}_{v}(3 n)\right) \leq e^{-1}
$$

Suppose next that $v \in V_{2}$. It follows from Lemmas 4 and 5 that we can find $w \in V_{1}$ such that $\operatorname{dist}(v, w) \leq \omega$. So from (35), with $\nu=3 n+\omega$, we have

$$
\operatorname{Pr}\left(\mathcal{A}_{v}(\nu)\right) \leq 1-\left(1-e^{-1}\right)(r s)^{-\omega},
$$

since if our walk visits $w$, it will with probability at least $(r s)^{-\omega}$ visit $v$ within the next $\omega$ steps. Thus if $\zeta=\left(1-e^{-1}\right)(r s)^{-\omega}$,

$$
\begin{aligned}
\sum_{\sigma \geq t_{0}} \operatorname{Pr}\left(\mathcal{A}_{v}(\sigma)\right) & \leq \sum_{\sigma \geq t_{0}}(1-\zeta)^{\lfloor\sigma / \nu\rfloor} \\
& \leq \sum_{\sigma \geq t_{0}}(1-\zeta)^{\sigma /(2 \nu)} \\
& =\frac{(1-\zeta)^{t_{0} /(2 \nu)}}{1-(1-\zeta)^{1 /(2 \nu)}} \\
& \leq 3 \nu \zeta^{-1}
\end{aligned}
$$


Thus, for all $u \in V$,

$$
\begin{aligned}
C_{u} & \leq t_{0}+5\left|V_{1}\right|+3\left|V_{2}\right| \nu \zeta^{-1} \\
& =t_{0}+O\left((r s)^{4 \omega} n\right) \\
& =t_{0}+o\left(t_{0}\right)
\end{aligned}
$$

as $\omega=\log \log \log n$.

\subsection{Lower bound on cover time}

For any vertex $u$, we can find a set of vertices $S$, such that at time $t_{1}=t_{0}(1-o(1))$, the probability the set $S$ is covered by the walk $\mathcal{W}_{u}$ tends to zero. Hence $T_{G}(u)>t_{1}$ whp which implies that $C_{G} \geq t_{0}-o\left(t_{0}\right)$.

We construct $S$ as follows. Let $S \subseteq V_{1}$ be some maximal set of locally tree-like vertices all of which are at least distance $2 \omega+1$ apart. Thus $|S| \geq\left(n-(r s)^{3 \omega}\right)(r s)^{-(2 \omega+1)}$.

Let $S(t)$ denote the subset of $S$ which has not been visited by $\mathcal{W}_{u}$ in the interval $[T, t]$. Now,

$$
\mathbf{E}|S(t)| \geq(1-o(1)) \sum_{v \in S}\left(\frac{1+o(1)}{\left(1+p_{v}\right)^{t}}+o\left(n^{-2}\right)\right) .
$$

Setting $t=t_{1}=(1-\epsilon) t_{0}$ where $\epsilon=2 \omega^{-1}$, we have

$$
\begin{aligned}
\mathbf{E}\left|S\left(t_{1}\right)\right| & =(1+o(1))|S| e^{-(1-\epsilon) t_{0} p_{v}} \\
& =(1+o(1)) \frac{n^{2 / \omega}}{(r s)^{2 \omega+1}} \\
& \geq n^{1 / \omega} .
\end{aligned}
$$

Let $Y_{v, t}$ be the indicator for the event that $\mathcal{W}_{u}$ has not visited vertex $v$ at time $t$. Let $Z=\{v, w\} \subset S$. We will show (below) that that for $v, w \in S$

$$
\mathbf{E}\left(Y_{v, t_{1}} Y_{w, t_{1}}\right)=\frac{1+o(1)}{\left(1+p_{Z}\right)^{t+2}}+o\left(n^{-2}\right),
$$

where $p_{Z} \sim p_{v}+p_{w}$. Thus

$$
\mathbf{E}\left(Y_{v, t_{1}} Y_{w, t_{1}}\right)=(1+o(1)) \mathbf{E}\left(Y_{v, t_{1}}\right) \mathbf{E}\left(Y_{w, t_{1}}\right) .
$$

It follows from (38) and (40), that

$$
\operatorname{Pr}\left(\left|S\left(t_{1}\right)\right|>T\right) \geq \frac{\left(\mathbf{E}\left|S\left(t_{1}\right)\right|-T\right)^{2}}{\mathbf{E}\left(\left(\left|S\left(t_{1}\right)\right|-T\right)^{2}\right)}=\frac{1}{\frac{\mathbf{E}\left(\left(\left|S_{t_{1}}\right|-T\right)\left(\left|S_{t_{1}}\right|-T-1\right)\right)}{\left(\mathbf{E}\left|S\left(t_{1}\right)\right|-T\right)^{2}}+\left(\mathbf{E}\left|S_{t_{1}}\right|-T\right)^{-1}}=1-o(1) .
$$


At most $T / \omega$ of $S\left(t_{1}\right)$ can be visited in the first $T$ steps and the lower bound follows.

Proof of (39). Let $G^{*}$ be obtained from $G$ by merging $v, w$ into a single node $Z$. This node has degree $2 r(s-1)$ and every other node has degree $r(s-1)$.

There is a natural measure preserving mapping from the set of walks in $G$ which start at $u$ and do not visit $v$ or $w$, to the corresponding set of walks in $G^{*}$ which do not visit $Z$. Thus the probability that $\mathcal{W}_{u}$ does not visit $v$ or $w$ during $[T, t]$ is equal to the probability that a random walk $\widehat{\mathcal{W}}_{u}$ in $G^{*}$ which also starts at $u$ does not visit $Z$ in the first $t$ steps.

We apply Corollary 2 to $G^{*}$. That $\pi_{Z}=\frac{2}{n}$ is clear. The derivation of $R_{T}(1)$ in Section 3.1 is also valid. The vertex $Z$ is tree-like up to distance $\omega$ in $G^{*}$. The fact that the root vertex of the corresponding infinite structure is in $2 r$ cliques does not affect the calculation of $R_{T}(1)$.

\section{Computation of $I(H)$ and $C_{E}(H)$}

This is very similar to the previous sections 4.1, 4.2 and so we will be light on details.

We briefly outline the upper bound proof for $I(H)$. Let $t_{0}=(1+o(1))\left(1+\frac{s-1}{(r-1)(s-1)-1}\right) \frac{n}{s-1} \log n$. Let $I_{u}(H)$ be the expected time for $\mathcal{W}_{u}$ to inform all vertices. Then

$$
I_{u}(H) \leq t+\sum_{v \in V} \sum_{\sigma \geq t} \operatorname{Pr}\left(\mathcal{B}_{v}(\sigma)\right)
$$

where $\mathcal{B}_{v}(\sigma)$ is the event that vertex $v$ is not informed in the interval $[T, \sigma]$.

For locally-tree-like vertices we use $p_{\gamma}$ from (25), and apply Corollary 2. For non-locally-treelike vertices we use the argument for (36) and obtain

$$
I_{u}(H) \leq t_{0}+5\left|V_{1}\right|+3\left|V_{2}\right| \nu \zeta^{-1}=t_{0}+o\left(t_{0}\right)
$$

as we did for (37).

We briefly outline the upper bound proof for $C_{E}(H)$. Let $t_{0}=(1+o(1))\left(1+\frac{s-1}{(r-1)(s-1)-1}\right) \frac{2 r n+s}{2 s} \log n$. Let $C_{E, u}(H)$ be the expected time for $\mathcal{W}_{u}$ to cover all edges. Then

$$
C_{E, u}(H) \leq t+\sum_{e \in E} \sum_{\sigma \geq t} \operatorname{Pr}\left(\mathcal{B}_{e}(\sigma)\right)
$$

where $\mathcal{B}_{e}(\sigma)$ is the event that vertex $e$ is not covered in the interval $[T, \sigma]$.

For locally-tree-like edges we use $p_{\gamma}$ from (26), and apply Corollary 2. For non-locally-tree-like vertices we use the argument for (36) and obtain as we did for (37),

$$
I_{u}(H) \leq t_{0}+5\left|E_{1}\right|+3\left|E_{2}\right| \nu \zeta^{-1}=t_{0}+o\left(t_{0}\right)
$$


where $E=E_{1} \cup E_{2}$ is a partition of $E$ into locally-tree-like edges and the rest.

\section{References}

[1] D. Aldous and J. Fill. Reversible Markov Chains andRandom Walks on Graphs. http://stat-wWw. berkeley.edu/pub/users/ aldous/RWG/book.html.

[2] R. Aleliunas, R.M. Karp, R.J. Lipton, L. Lovász and C. Rackoff, Random Walks, Universal Traversal Sequences, and the Complexity of Maze Problems. Proceedings of the 20th Annual IEEE Symposium on Foundations of Computer Science (1979) 218-223.

[3] C. Avin, Y. Lando, and Z. Lotker. Simple Random Walks on Radio Networks. http://arxiv.org/abs/0907.1678.

[4] B.Bollobás, A probabilistic proof of an asymptotic formula for the number of labelled regular graphs, European Journal on Combinatorics 1 (1980) 311-316.

[5] J. Cong, L. Hagen and A. Kahng. Random walks for circuit clustering. Proc. 4th IEEE Intl. ASIC Conf., (1991) 14.2.1 - 14.2.4.

[6] C. Cooper and A. M. Frieze, The cover time of random regular graphs, SIAM Journal on Discrete Mathematics, 18 (2005) 728-740.

[7] C. Cooper and A. M. Frieze, The cover time of the giant component of of $G_{n, p}$. Random Structures and Algorithms, 32, 401-439 J. Wiley (2008).

[8] W. Feller, An Introduction to Probability Theory, Volume I, (Second edition) Wiley (1960).

[9] M. Jerrum and A. Sinclair. The Markov chain Monte Carlo method: an approach to approximate counting and integration. In Approximation Algorithms for NTP-hard Problems. (D. Hochbaum ed.) PWS (1996) 482-520

[10] L. Lovász. Random walks on graphs: A survey. Bolyai Society Mathematical Studies, 2:353-397, Budapest, 1996.

[11] D. Zhou, J, Huang and B. Schölkopf. Learning with Hypergraphs: Clustering, Classifcation, and Embedding. Advances in Neural Information Processing Systems (NIPS) 19 1601-1608. (Eds.) B. Schölkopf, J.C. Platt and T. Hofmann, MIT Press, Cambridge, MA, 2007. 